\title{
Influência de parâmetros da estimulação elétrica funcional na contração concêntrica do quadríceps
}

\author{
Ingrid Johanna Rodríguez Bohórquez*, Marcio Nogueira de Souza, Alexandre Visintainer Pino
}

Resumo Introdução: Sistemas de estimulação elétrica funcional (FES) são usados na prática clínica para o fortalecimento muscular, assim como para a recuperação e preservação funcional dos músculos enfraquecidos. Porém, uma das dificuldades encontradas na sua aplicação é a falta de padrões adequados para a realização de um movimento pré-estabelecido, uma vez que os parâmetros da estimulação (frequência, intensidade de corrente e duração do pulso) são variados heuristicamente. O presente trabalho investigou a influência dos parâmetros da FES usada no estabelecimento da posição angular e força produzida na extensão de joelho. Métodos: $\mathrm{O}$ procedimento experimental foi dividido em duas fases chamadas Fase I e Fase II. A amostra total do estudo foi composta por 41 voluntários saudáveis de ambos os sexos (27 na Fase I e 14 na Fase II). Durante a Fase I cada voluntário foi estimulado com 18 combinações diferentes de parâmetros para a FES, com o objetivo de determinar um modelo capaz de prever o movimento realizado para cada padrão de estimulação. Resultados: As diferentes durações de pulso aplicadas não resultaram em alterações significativas nos movimentos produzidos. Dentre os valores de frequência investigados, $50 \mathrm{~Hz}$ foi escolhida para a Fase II, por ter apresentado maior diferença estatisticamente significativa para os valores de intensidade de corrente avaliados. Durante a Fase II, a intensidade de corrente da FES foi ajustada segundo um modelo linear estabelecido durante a primeira fase e que considerou características antropométricas dos sujeitos; encontrando-se um coeficiente de correlação $r=0,798$ entre o ângulo pré-estabelecido e o ângulo alcançado. Conclusão: Este trabalho apresenta um modelo para a determinação de parâmetros de FES que permitem estabelecer variações angulares da articulação do joelho associadas à eletroestimulação. Considerando a frequência e a duração de pulsos constantes, se estima, usando uma equação de primeira ordem e com coeficiente de correlação em torno de 0,72, a intensidade de corrente dependente da variação de angulação desejada

Palavras-chave Estimulação elétrica funcional FES, Variação angular, Força muscular.

\section{Influence of functional electrical stimulation parameters in quadriceps concentric contraction}

\begin{abstract}
Introduction: Functional electrical stimulation (FES) is used in clinical practice for muscle strengthening, as well as for functional recovery and preservation of weakened muscles. However, one of the problems in clinical application is the lack of adequate standards for determining the attainment of a predetermined movement, since the stimulation parameters are often adjusted heuristically. The present study investigated the influence of some parameters (frequency, current intensity and pulse duration) of the FES used to establish the joint angle and force produced in knee extension. Methods: The experimental procedure was divided in two phases (Phase I and Phase II). The total sample included 41 healthy volunteers of both sexes (27 and 14 in Phase I and Phase II). During Phase I 18 profiles of FES were applied in the quadriceps muscle of subjects, changing the above-mentioned FES parameters. Results: It was observed that the pulse duration of the electrical stimulation pulse did not exhibit significant relevance in the evoked movements. Among the discrete values of frequency investigated, the stimulation at $50 \mathrm{~Hz}$ was selected because it showed higher statistical significant difference considering the values of current intensity measured. During the Phase II, devoted to place the knee joint angle in a pre-determined value, the current intensity of the FES was adjusted according to the linear model established during the Phase I, which considered anthropometric characteristics of the subjects. In this Phase we found a correlation coefficient $r=0,798$ between the pre-determined angle and the reached one. Conclusion: This paper presents a model for the determination of FES parameters for establishing angular variations of the knee associated with electrical stimulation. Considering frequency and pulse duration constants we estimate, using a first order equation and correlation coefficient of 0.72 , the current intensity dependent of desired angle variation.
\end{abstract}

Keywords Functional Electrical Stimulation FES, Angular variation, Muscle force.

*e-mail: ingridr@peb.ufrj.br

Recebido: 19/05/2012/ Aceito: 01/02/2013 


\section{Introdução}

A estimulação elétrica funcional (FES - Functional Electrical Stimulation), pode ser definida como uma técnica de estimulação elétrica dos neurônios motores com a finalide de produzir contração muscular. $\mathrm{O}$ propósito da FES é o movimento ou alinhamento postural funcional (Binder-Macleod e Lee, 1997; Kitchen, 2003; Singer, 1987).

Sistemas de FES são usados na prática clínica para o fortalecimento do músculo enfraquecido e a recuperação ou preservação da função do mesmo durante a fase de atividade reduzida ou de imobilização (Maffiuletti, 2010). Além disso, também tem sido relatada a ocorrência de adaptações neurais associadas ao uso da eletroestimulação (Braz et al., 2009), sendo um processo importante para a retomada de interação do paciente com o ambiente (Peckham e Knutson, 2005; Sinkjaer e Popovic, 2009).

A principal dificuldade encontrada na aplicação clínica da FES é a falta de padrões adequados de estimulação, em particular da amplitude de corrente, para a realização de um movimento pré-estabelecido. Verifica-se, então, a necessidade de desenvolvimento de modelos que descrevam a os efeitos da contração muscular em resposta à estimulação elétrica (Pedotti e Ferrarin, 1992; Perumal et al., 2006). Estes modelos poderiam auxiliar na previsão dos movimentos produzidos para um dado padrão de estimulação elétrica muscular, evitando-se ou reduzindo-se experiências cansativas ou procedimentos errôneos para o paciente em reabilitação.

Apresentam-se na literatura modelos mecânicos da variação angular, ou força resultante, da articulação do joelho devido à estimulação dos músculos quadríceps (El Makssoud et al., 2003; Ferrarin e Pedotti, 2000; Franken et al., 1993; Lynch et al., 2011; Lynch e Popovic, 2011; Mohammed et al., 2010; Perumal et al., 2006) e também, mesmo que em menor quantidade, estudos sobre a influência de medidas antropométricas sobre os efeitos da FES (Doheny et al., 2010; Gave et al., 2011; Maffiuletti, 2010; Maffiuletti et al., 2011; Petrofsky, 2008). Contudo, não é possível medir todas as variáveis que determinam exatamente como um músculo irá responder à estimulação elétrica, de modo que as variações na resposta do músculo não têm sido padronizadas. Deste modo, existe carência de modelos que usem parâmetros facilmente determináveis e que sejam adequados para aplicações clínicas.

O presente trabalho tem como objetivo principal apresentar os resultados da avaliação da influência de alguns parâmetros da FES (frequência, intensidade de corrente e duração do pulso) na determinação da posição angular e força produzida no movimento de extensão da articulação do joelho. O trabalho pretende contribuir na padronização de parâmetros de estimulação elétrica fisioterápica, com base nas características individuais dos sujeitos.

\section{Métodos}

O procedimento experimental foi dividido em Fase I e Fase II, descritas mais adiante. A amostra para ambas as fases foi composta por 41 voluntários saudáveis (26 homens, 15 mulheres) de ambos os sexos com idade mínima de 18 anos, cujas características gerais podem ser vistas na Tabela 1 . Todos os voluntários receberam informação sobre o objetivo e os procedimentos que seriam realizados, concordaram e assinaram os termos de consentimento para a participação no estudo, aprovado pelo Comitê de ética e pesquisa institucional do Hospital Universitário Clementino Fraga Filho da Faculdade de Medicina (CEP-HUCFF/ FM) sob número CEP 003/11.

Os critérios de inclusão contemplaram sujeitos que não apresentassem histórico de alterações ou lesões do sistema neuromuscular e nenhum objeto metálico implantado nos membros inferiores, que conseguissem realizar o movimento de flexão e extensão do joelho em toda sua amplitude, e que não tivessem nenhuma lesão de pele na região onde foram colocados os eletrodos. Os critérios de exclusão das duas fases são todos aqueles que não se enquadram nos critérios de inclusão.

\section{Avaliação antropométrica}

Durante ambas as fases foram realizadas medições antropométricas dos sujeitos segundo o protocolo de Guedes (2006), onde foram obtidas informações sobre a estatura, massa corporal, perímetro da coxa $(C c x)$, da perna medial, espessura das dobras cutâneas da coxa $(D C c x)$, comprimento da coxa, da perna e o comprimento total do membro inferior direito. Todas as medidas foram realizadas três vezes consecutivas, considerando-se o valor médio da série como a medida representativa para análise e estimação de massas de alguns segmentos corporais a partir de equações propostas na literatura (Enoka, 1994).

Adicionalmente foram estimadas a área transversa da coxa $(A T C x)$, a área muscular da coxa $(A M c x)$, a área de gordura da coxa $(A G c x)$ e volume da coxa (Volcx), apresentadas nas Equações 1, 2, 3 e 4 (onde $L$ é o comprimento do segmento perna-pé em metros).

$A T c x=\frac{C c x^{2}}{4 \times \pi}$ 
Tabela 1. Características gerais dos voluntários (média + desvio padrão).

Table 1. Volunteers characteristics (mean + standart deviation).

\begin{tabular}{|c|c|c|}
\hline & Fase I & Fase II \\
\hline & Homens $(n=18)$ & Homens $(\mathrm{n}=8)$ \\
\hline & Mulheres $(n=9)$ & Mulheres $(n=6)$ \\
\hline \multirow[t]{2}{*}{ Idade (anos) } & $24,1+5,2$ & $29,4+3,5$ \\
\hline & $28,1+9,2$ & $29,2+4,1$ \\
\hline \multirow[t]{2}{*}{ Altura $(\mathrm{cm})$} & $174,4+5,7$ & $176,2+5,2$ \\
\hline & $161,6+6,9$ & $160,8+4,1$ \\
\hline \multirow[t]{2}{*}{ Massa corporal $(\mathrm{kg})$} & $79,3+12,0$ & $83,4+5,7$ \\
\hline & $57,8+8,2$ & $60,2+13,6$ \\
\hline \multirow[t]{2}{*}{ Comprimento membro inferior $(\mathrm{cm})$} & $88,8+3,6$ & $91,3+3,0$ \\
\hline & $86,4+3,9$ & $87,6+2,3$ \\
\hline \multirow[t]{2}{*}{ Comprimento coxa $(\mathrm{cm})$} & $39,1+2,4$ & $40,1+2,5$ \\
\hline & $39,8+1,8$ & $41,5+2,7$ \\
\hline \multirow[t]{2}{*}{ Comprimento perna $(\mathrm{cm})$} & $43,7+5,4$ & $41,4+1,5$ \\
\hline & $40,8+3,9$ & $35,9+2,7$ \\
\hline \multirow[t]{2}{*}{ Comprimento perna + pé $(\mathrm{cm})$} & $62,6+2,1$ & $62,2+2,0$ \\
\hline & $57,0+4,0$ & $56,7+1,9$ \\
\hline \multirow[t]{2}{*}{ Perímetro da coxa $(\mathrm{cm})$} & $55,2+4,0$ & $56,7+3,4$ \\
\hline & $52,0+4,5$ & $52,0+5,6$ \\
\hline \multirow[t]{2}{*}{ Perímetro da perna medial $(\mathrm{cm})$} & $39,8+5,1$ & $38,4+1,1$ \\
\hline & $35,4+2,6$ & $35,9+2,7$ \\
\hline \multirow[t]{2}{*}{ Dobra cutânea $(\mathrm{cm})$} & $1,9+0,8$ & $1,4+0,4$ \\
\hline & $2,7+0,4$ & $2,2+1,2$ \\
\hline \multirow[t]{2}{*}{ Área total da coxa $\left(\mathrm{cm}^{2}\right)$} & $244,1+34,8$ & $257,1+30,5$ \\
\hline & $216,5+38,1$ & $217,3+47,1$ \\
\hline \multirow[t]{2}{*}{ Área muscular $\left(\mathrm{cm}^{2}\right)$} & $192,9+26,9$ & $217,5+25,8$ \\
\hline & $152,1+29,9$ & $166,8+66,7$ \\
\hline \multirow[t]{2}{*}{ Área de gordura $\left(\mathrm{cm}^{2}\right)$} & $51,2+22,3$ & $39,6+11,4$ \\
\hline & $64,5+12,6$ & $50,4+28,2$ \\
\hline \multirow[t]{2}{*}{ Volume da coxa $\left(\mathrm{cm}^{3}\right)$} & $9573,3+1648,4$ & $10315,0+1426,0$ \\
\hline & $8623,7+1583,4$ & $9069,4+2204,2$ \\
\hline
\end{tabular}

$A M c x=\frac{[C c x-(D C c x \times \pi)]^{2}}{4 \times \pi}$

$A G c x=A T c x-A M c x$

Volcx $=A T c x \times L$

\section{Modelo biomecânico}

Foram realizadas estimativas da força muscular na contração concêntrica do quadríceps (a contração vence a resistência e há o encurtamento muscular durante a geração de força) propiciada pela FES. Nestas estimativas os segmentos corporais envolvidos foram considerados barras de densidade uniforme e o braço de alavanca constante (Figura 1).

Para o modelo biomecânico desenvolvido, o segmento perna-pé, que roda em torno de seu eixo articular, possui momento de inércia dado por (Equação 5):

$I=\frac{1}{3} m L^{2}$ onde $L$ é o comprimento do segmento em metros e $m$ é a massa do segmento em quilogramas.

A massa do segmento perna-pé foi calculada a partir de equações reportadas na literatura (Equação 6), onde $m_{c}$ é a massa corporal (Enoka, 1994):

$m=0,053 m_{c}+0,73$

A partir da aquisição da evolução temporal da angulação, da articulação do joelho durante a eletroestimulação, foi obtida por derivação numérica a velocidade e a aceleração angular. Posteriormente se fez a identificação dos máximos da aceleração.

Com estes dados foi estimada a força realizada durante a aceleração máxima do segmento (Equação 7).

$|F m|=\frac{I|\ddot{\theta}|+m g l \sin \sin (\theta)}{d}$

onde o ângulo $\theta$ é definido como o complemento da variação angular associada à FES, $\ddot{\theta}$ é a aceleração angular (obtida numericamente), $I$ é o momento de inércia do segmento perna-pé, $m g$ é o peso do segmento, $d$ é o raio do joelho, cujo valor sugerido na 
literatura é de 0,04 m (Tozeren, 2000), e $l$ é a distância do centro de gravidade do segmento à articulação do joelho $(0,434 \cdot L)$, dada em metros (Enoka, 1994).

Para cada perfil de estimulação foi calculada a média da força muscular, obtida pela Equação 7, cujo valor foi considerado para análise estatística.

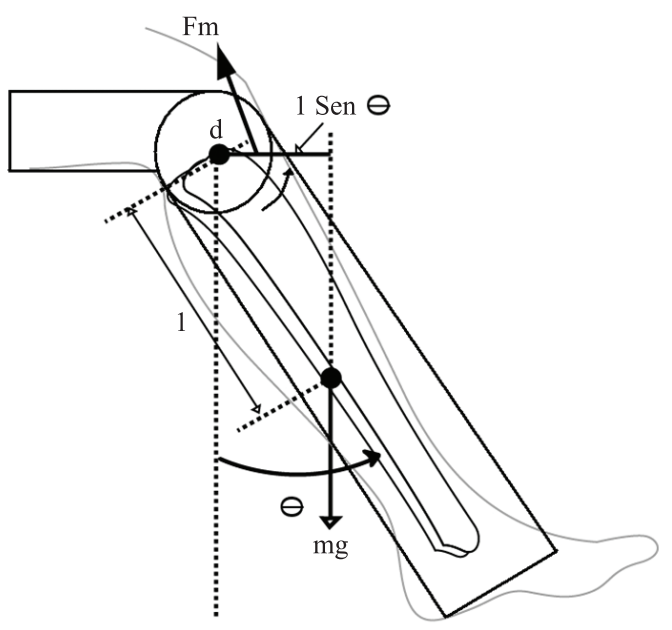

Figura 1. Modelo biomecânico para a estimativa da força $(\mathrm{Fm})$ exercida pelo grupo muscular do quadríceps durante o movimento de extensão de joelho, onde o ângulo $\theta$ é definido como o complemento da variação angular associada à FES, $m g$ é o peso do segmento, $d$ é o raio do joelho e $l$ é a distância do centro de gravidade do segmento à articulação do joelho.

Figure 1. Biomechanical model to estimate the force (Fm) applied by the quadriceps muscle group during movement of knee extension, where the angle $\theta$ is defined as the complement of the angular variation associated with FES, $m g$ is the weight of the segment, $d$ is the knee's radius and lis the distance from the center of gravity of the segment to the knee joint.
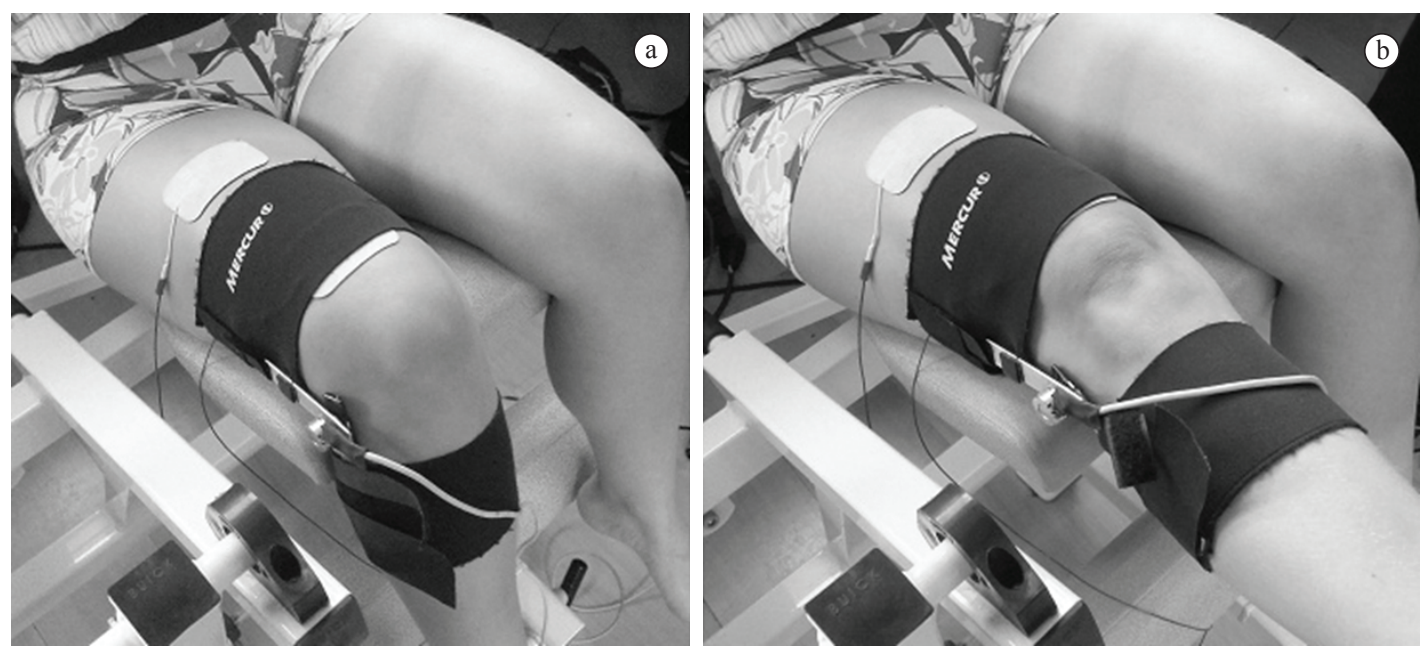

Figura 2. Eletrogoniômetro para medição da variação angular: a) Posição de descanso, b) Extensão do joelho.

Figure 2. Electrogoniometer for measuring the angular variation: a) Rest position, b) Knee joint extension.

\section{Protocolo de estimulação}

Os voluntários se sentaram numa cadeira adaptada com o quadril e o joelho flexionados em ângulo de $80^{\circ}$ (posição de descanso). Após a limpeza da pele, dois eletrodos auto-adesivos de $5 \times 9 \mathrm{~cm}$ (Axelgaard, modelo ValuTrode CF5090, EUA) foram posicionados sobre a coxa, sendo o anodo posicionado na região supra-patelar (4 $\mathrm{cm}$ da borda superior da patela). Para posicionar o catodo sobre a região do triângulo femoral realizou-se um teste para localização do ponto motor. Neste teste foram utilizados pulsos de corrente de 5 a $20 \mathrm{~mA}$, com duração de pulso de $300 \mu$ s e frequência de $1 \mathrm{~Hz}$, aplicados por meio de uma caneta de eletroestimulação, até se gerar abalo muscular no ponto motor do vasto lateral. A região na qual se notava forte contração muscular era marcada para posicionar o catodo.

Um eletrogoniômetro potenciométrico foi posicionado lateralmente ao joelho para medir a variação do ângulo associada ao movimento de extensão do joelho (Figura 2).

\section{Estimulação elétrica}

Na Fase I realizaram-se 18 combinações diferentes de parâmetros da FES em seis sessões de estimulação, cada uma com três perfis (combinações). Cada perfil de estimulação manteve constante a frequência do trem de pulsos em $30 \mathrm{~Hz}, 50 \mathrm{~Hz}$ ou $80 \mathrm{~Hz}$, e a duração dos pulsos em $300 \mu \mathrm{s}$ ou $400 \mu \mathrm{s}$. A intensidade de corrente (i) aumentada linearmente ao longo do tempo desde $0 \mathrm{~mA}$, apresentando valores máximos de $40 \mathrm{~mA}, 50 \mathrm{~mA}$ ou $60 \mathrm{~mA}$ (Figura 3). Para a contração excêntrica do quadríceps (a resistência vence a contração havendo o alongamento muscular, o músculo age desacelerando 


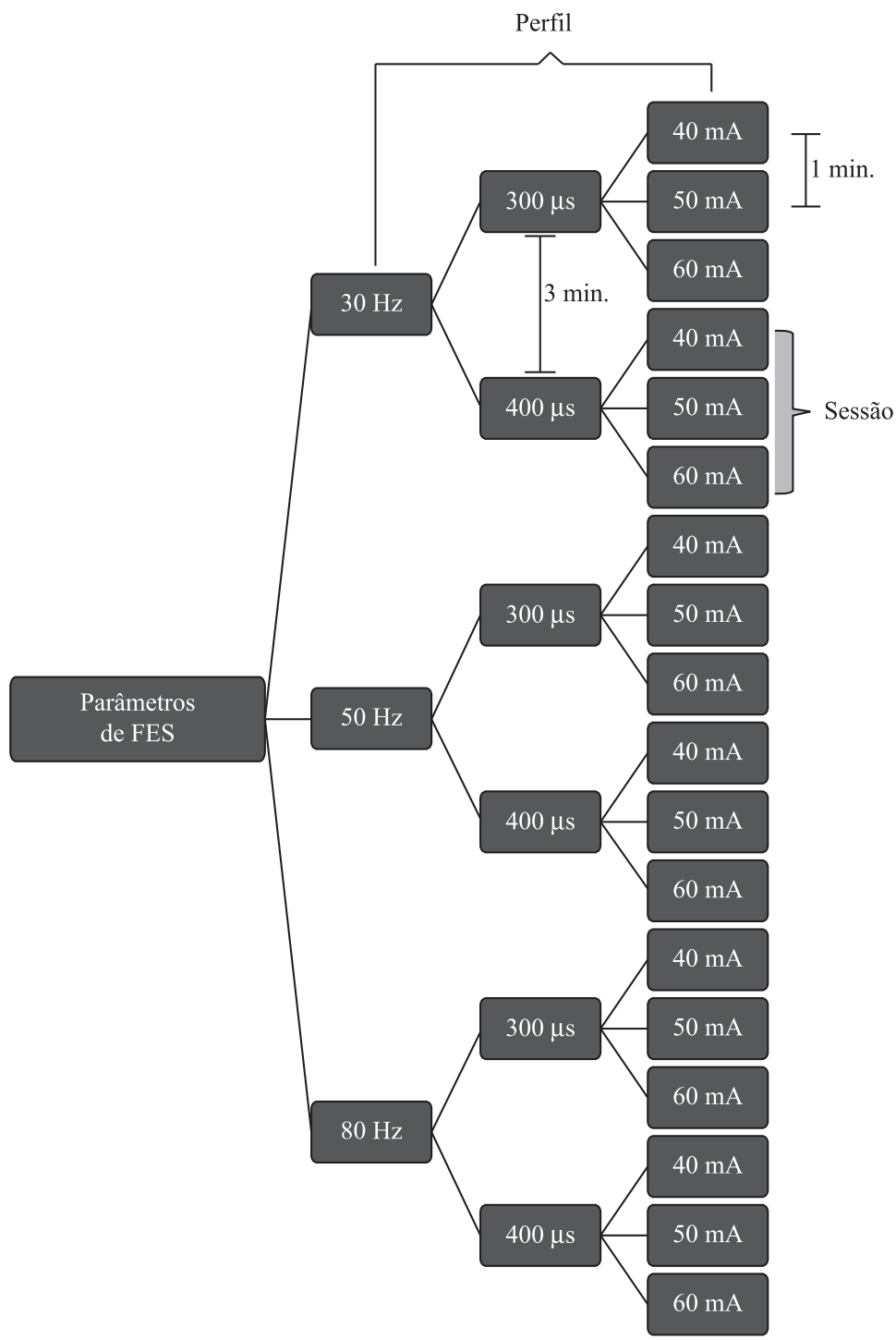

Figura 3. Perfis de estimulação do protocolo experimental da Fase I. Figure 3. FES Profiles for the experimental protocol of Phase I.

o movimento de forma controlada) após a aplicação da máxima corrente esta foi diminuída de forma simétrica (Figura 4).

Os perfis de corrente de estimulação que, eventualmente, provocassem sensação de dor eram descartados. Durante os experimentos os voluntários mantiveram na mão o controle para desligar a estimulação.

Para cada perfil de parâmetros da FES foram efetuadas seis repetições do movimento de contração concêntrica do quadríceps com intervalo de repouso de 3 segundos entre elas. $\mathrm{O}$ intervalo de repouso entre perfis diferentes foi de 1 minuto. Considerou-se uma sessão, o conjunto de perfis que mantiveram fixas a frequência e duração de pulsos, variando-se somente a intensidade. $\mathrm{O}$ intervalo de repouso entre as sessões foi de 3 minutos.

$\mathrm{Na}$ fase I foram avaliadas a posição angular medida (AngMed) e a força máxima estimada $(F m)$ para cada um dos 18 perfis de estimulação.

Na Fase II, realizou-se uma sessão de estimulação, durante a qual se manteve fixas a frequência e a duração de pulsos nos valores obtidos na avaliação das medidas da posição angular, Fase I. Variou-se a intensidade de corrente, com a intenção de posicionar a articulação do joelho nos ângulos de 15, 30 e $45^{\circ}$.

O equipamento utilizado para a geração dos pulsos de estimulação foi o estimulador FES-PEB (Velloso, 


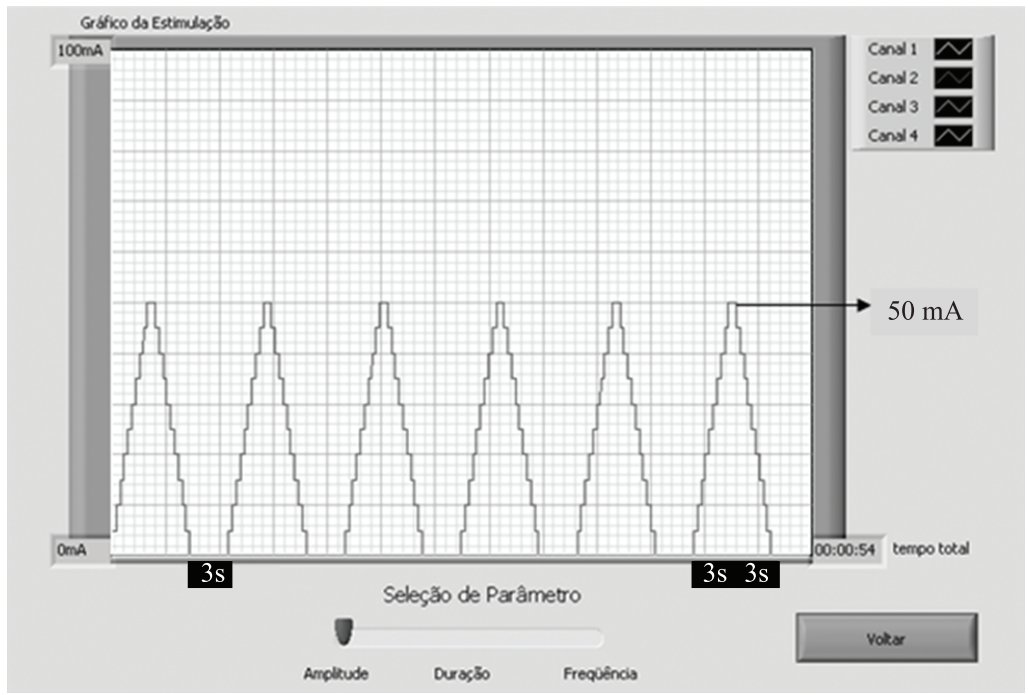

Figura 4. Exemplo de perfil de FES criado no programa de interface com o usuário do equipamento FES-PEB.

Figure 4. Example of FES profile created with the user interface of the FES-PEB equipment.

2007), o qual fornece intensidade de corrente entre 0 e $100 \mathrm{~mA}$, com resolução de $1 \mathrm{~mA}$, duração entre 50 e $500 \mu$ s, com resolução de $1 \mu$ s, e com frequência entre 20 e $200 \mathrm{~Hz}$, com resolução de $1 \mathrm{~Hz}$.

\section{Aquisição de sinais e sensores}

A coleta dos dados de corrente de estimulação e de goniometria foi realizada utilizando a placa de aquisição NI USB-6251 da National Instruments (16 bits, a uma frequência de amostragem de $23900 \mathrm{~Hz}$ ). Para a aquisição foi utilizado o programa DAS (Pino et al., 2004), desenvolvido em LabVIEW 7.1. Deve ser mencionado que a corrente de estimulação foi digitalizada a partir um amostrador de corrente colocado em série com o circuito de estimulação (Gave et al., 2011).

A amplitude da angulação articular foi digitalizada a partir da saída de um eletrogoniômetro. Este goniômetro foi calibrado de modo a fornecer uma saída entre 0 e $135^{\circ}$.

O processamento off-line dos sinais coletados foi realizado no programa Mecanica (Pino et al., 2002). Para cada repetição do movimento foram determinados os ângulos iniciais e finais da articulação do joelho e o valor máximo da corrente de excitação. Para cada perfil foi calculada a média da variação angular, que corresponde à diferença entre o ângulo final e o inicial do sinal de goniometria.

Descartaram-se sinais que mostrassem um visível comportamento de contração voluntária do sujeito durante o período de aplicação da FES, assim como alterações permanentes na linha de base (sinal do goniômetro não retornando ao ângulo articular inicial).

\section{Análise estatistica}

A análise estatística dos dados foi feita no software R 2.13.0. (Ihaka e Gentleman, 1996). Os dados experimentais relativos às seis repetições de movimento foram inicialmente descritos por média e desvio padrão. No primeiro momento foi elaborada uma análise exploratória entre as variáveis dependentes (AngMed e $\mathrm{Fm}$ ) e as variáveis independentes (frequência, duração de pulso e intensidade de corrente).

Aos dados experimentais de AngMed e Fm da Fase I aplicou-se o teste de Wilcoxon para avaliar a influência da largura de pulso sobre estas grandezas em todas as combinações de corrente e frequência. O teste de Kruskal-Wallis foi usado para investigar a influência de frequência de estimulação sobre AngMed e Fm para cada valor de corrente. Ambos os testes foram feitos ao nível de significância de 0,05 e com ajuste dos valores $p$ pelo método de Bonferroni. Com a largura de pulso e frequência que produziram as maiores angulações e forças foram analisadas a influência das variáveis antropométricas sobre o movimento.

Foi avaliada a normalização dos valores das variações angulares em função da corrente (i) conforme Equação 8.

AngMed $/ \overline{\text { AngMed }}=a i+b \mid$ voluntário

onde $a$ e $b$ são constantes e $\overline{A n g M e d}$ é a média das seis variações angulares, considerando-se cada voluntário.

Também foram investigadas as influências das variáveis antropométricas estudadas como possíveis moduladoras da corrente (i) e seus efeitos sobre a 
AngMed e Fm segundo os modelos descritos pelas Equações 9 e 10, respectivamente.

$$
\begin{aligned}
& \text { AngMed }=a \frac{i}{\text { Norm }}+b \\
& F_{m}=a \frac{i}{\text { Norm }}+b
\end{aligned}
$$

onde $a$ e $b$, são constantes e Norm são os normalizadores: 1, Ccx, DCcx, ATcx, AGcx, Volcx.

$\mathrm{Na}$ Fase II, intensidades de corrente foram preditas pelos modelos desenvolvidos na Fase I (Equação 11), com o objetivo de posicionar a articulação do joelho em ângulos pré-determinados. Tais ângulos foram, então, confrontados com seus análogos reais adquiridos experimentalmente, calculando-se o coeficiente de correlação de Pearson para avaliar a associação entre as mesmas.

$i=\operatorname{Norm} \frac{\text { AngMed }-b}{a}$

\section{Resultados}

Os resultados da análise exploratória das variáveis de resposta (AngMed e Fm) mostraram uma distribuição assimétrica das mesmas. Por esta razão, foram utilizados testes não paramétricos neste trabalho. Não foram encontradas diferenças estatisticamente significativas na AngMed nem na Fm (Tabela 2) para as larguras de pulso analisadas.
Em decorrência disto, escolheu-se a largura de $300 \mu$ s para a Fase 2. No teste de Kruskal-Wallis, observou-se que na frequência de $50 \mathrm{~Hz}$ tanto a $\mathrm{AngMed}$ quanto a $\mathrm{Fm}$ apresentaram diferenças estatisticamente significativas para todas as correntes analisadas. O mesmo não ocorreu para a frequência de $30 \mathrm{~Hz}$. Observar também que há diferenças significativas para os efeitos da estimulação se a frequência é alterada de 30 para $50 \mathrm{~Hz}$, mas o mesmo não ocorre quando a frequência é aumentada para $80 \mathrm{~Hz}$ (Figura 5) como era esperado (Chou et al., 2005).

Em virtude destes achados todos os demais resultados apresentados nesta secção correspondem à análise de dados feita com estimulação de frequência e duração de pulso de, respectivamente, $50 \mathrm{~Hz}$ e $300 \mu$ s.

No modelo ajustado entre a intensidade de corrente e a angulação normalizada pela sua média, para cada sujeito, foi encontrado um coeficiente de correlação $(r)$ de 0,948 indicando que uma modelagem linear entre as duas variáveis é capaz de explicar aproximadamente $89 \%$ da variabilidade encontrada (Figura 6a). Para os modelos que descrevem a AngMed e a Fm em função da corrente, sem nenhum tipo de normalização característica de cada indivíduo o coeficiente de correlação linear entra as variáveis analisadas se mostrou muito menor $(0,701$ e 0,706 , Figura $6 b$, c, respectivamente). Isto indica que apenas cerca de $50 \%$ da variabilidade de $A n g M e d$ e $F m$ podem ser explicados pela corrente sem normalização de nenhum tipo.

Tabela 2. Test Wilcoxon entre as durações de pulso (média + desvio padrão) mantendo frequência e corrente constantes nas medições experimentais da Fase I.

Table 2. Values of Wilcoxon test (mean + standart deviation) keeping constant the frequency and current experimental measurements of phase I.

\begin{tabular}{|c|c|c|c|c|c|c|}
\hline & \multicolumn{3}{|c|}{ AngMed } & \multicolumn{3}{|c|}{$F m$} \\
\hline & $300 \mu \mathrm{s}$ & $400 \mu \mathrm{s}$ & Valor $p$ & $300 \mu \mathrm{s}$ & $400 \mu \mathrm{s}$ & Valor $p$ \\
\hline $30 \mathrm{~Hz}$ & $6,98+5,17$ & $9,49+9,84$ & 0,81 & $26,11+20,99$ & $31,14+25,24$ & 0,66 \\
\hline $50 \mathrm{~Hz}$ & $6,52+9,54$ & $10,60+13,56$ & 0,22 & $21,01+23,12$ & $32,11+30,82$ & 0,23 \\
\hline $80 \mathrm{~Hz}$ & $5,03+3,77$ & $10,36+10,54$ & 0,21 & $17,79+11,11$ & $32,21+24,65$ & 0,15 \\
\hline
\end{tabular}
$40 \mathrm{~mA}$

\begin{tabular}{|c|c|c|c|c|c|c|}
\hline & \multicolumn{6}{|c|}{$50 \mathrm{~mA}$} \\
\hline & \multicolumn{3}{|c|}{ AngMed } & \multicolumn{3}{|c|}{$F m$} \\
\hline & $300 \mu \mathrm{s}$ & $400 \mu \mathrm{s}$ & Valor $p$ & $300 \mu \mathrm{s}$ & $400 \mu \mathrm{s}$ & Valor $p$ \\
\hline $30 \mathrm{~Hz}$ & $22,67+18,94$ & $29,46+24,31$ & 0,45 & $71,87+43,72$ & $87,56+51,54$ & 0,33 \\
\hline $50 \mathrm{~Hz}$ & $22,31+20,45$ & $32,79+23,00$ & 0,087 & $72,22+48,51$ & $100,43+55,57$ & 0,095 \\
\hline \multirow[t]{4}{*}{$80 \mathrm{~Hz}$} & $22,79+17,85$ & $29,15+23,40$ & 0,46 & $74,20+43,18$ & $90,82+59,78$ & 0,38 \\
\hline & \multicolumn{6}{|c|}{$60 \mathrm{~mA}$} \\
\hline & \multicolumn{3}{|c|}{ AngMed } & \multicolumn{3}{|c|}{$F m$} \\
\hline & $300 \mu \mathrm{s}$ & $400 \mu \mathrm{s}$ & Valor $p$ & $300 \mu \mathrm{s}$ & $400 \mu \mathrm{s}$ & Valor $p$ \\
\hline $30 \mathrm{~Hz}$ & $34,65+22,12$ & $39,68+22,34$ & 0,49 & $104,42+46,63$ & $122,23+58,74$ & 0,12 \\
\hline $50 \mathrm{~Hz}$ & $41,02+21,44$ & $45,63+20,73$ & 0,38 & $124,86+46,17$ & $142,57+57,88$ & 0,2 \\
\hline $80 \mathrm{~Hz}$ & $37,80+23,09$ & $44,44+23,80$ & 0,26 & $120,27+59,02$ & $141,99+63,56$ & 0,16 \\
\hline
\end{tabular}



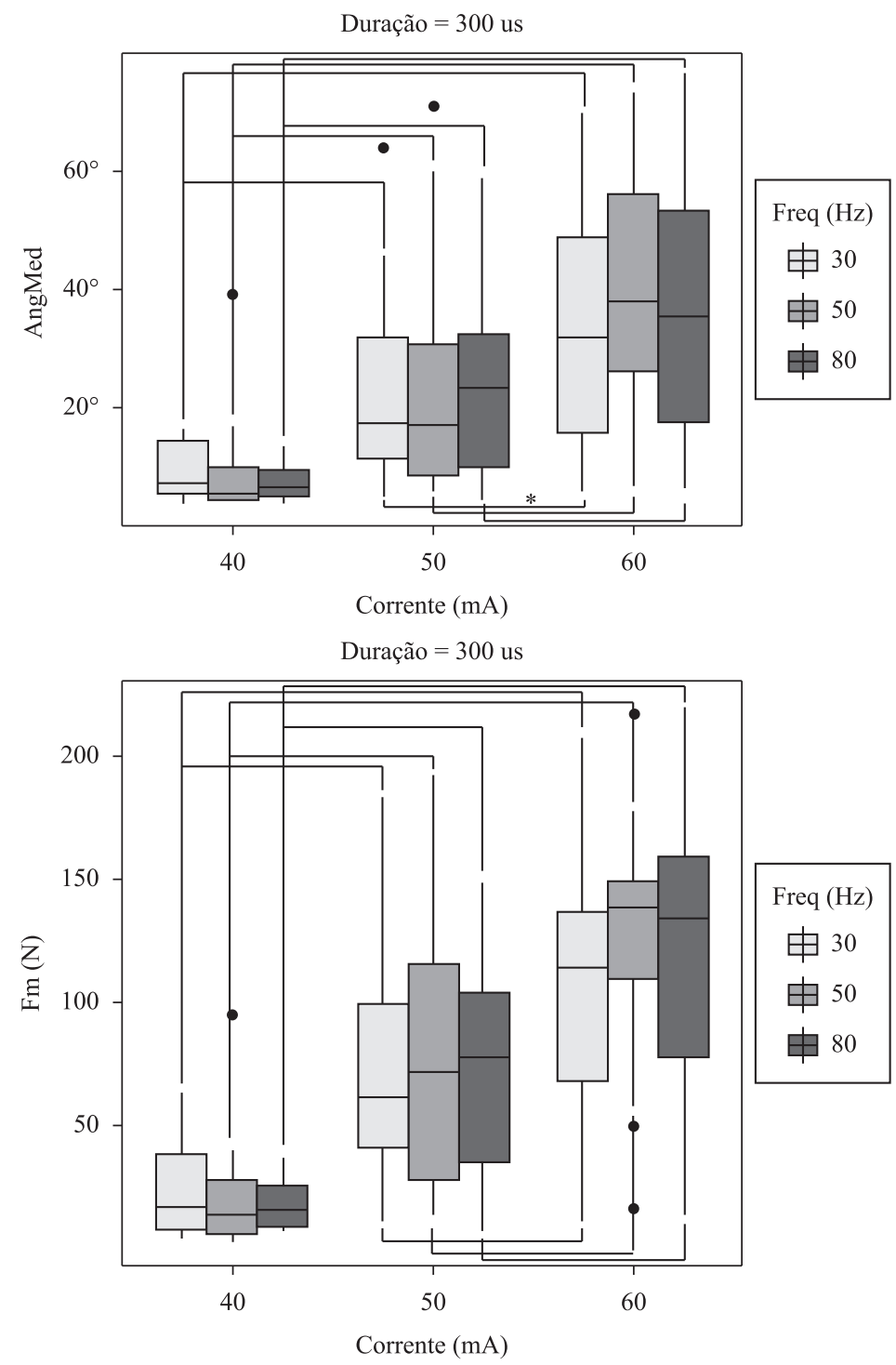

Figura 5. Box-plot das variáveis de resposta $($ AngMed e $F m)$ em função da intensidade de corrente. $\mathrm{O}$ asterisco $(*)$ indica que não há diferença estaticamente significativa entre as populações.

Figure 5. Box-plot of AngMed and Fm vs current. * meaning that there is no significant difference.

A busca por normalizadores antropométricos que pudessem descrever melhor o comportamento da AngMed e da Fm, em função das correntes aplicadas a cada indivíduo, resultou em coeficientes de correlação linear menores ou ligeiramente melhores do que os encontrados sem normalização. Os melhores resultados apareceram na estimativa de AngMed sendo o normalizador $C c x$ (Figura 7) aquele que apresentou os melhores resultados.

$\mathrm{Na}$ estimativa de AngMed os coeficientes de correlação encontrados para os normalizadores: $C c x$, $D C c x, A T c x, A G c x$, Volcx foram respectivamente $0,723,0,008,0,723,0,711$ e 0,679 . Cabe ressaltar que a inclusão de $C c x$ ou $A T c x$ como normalizadores resultou em uma melhora de aproximadamente 3\% na estimativa de AngMed e a inclusão de informação relativas as dobras cutâneas $(D C c x)$ resultou em uma correlação quase nula entre AngMed e a corrente normalizada.

Para a $F m$ os resultados foram semelhantes, porém não houve aumento no coeficiente de correlação entre $F m$ e a corrente normalizada. O uso dos normalizadores: $C c x, D C c x, A T c x, A G c x$, Volcx resultou correlações de $0,685,0,289,0,583,0,550$ e 0,580 respectivamente. Mais uma vez $C c x$ apresentou-se como o melhor normalizador dentre todos os avaliados e $D C c x$ como o pior de todos. 

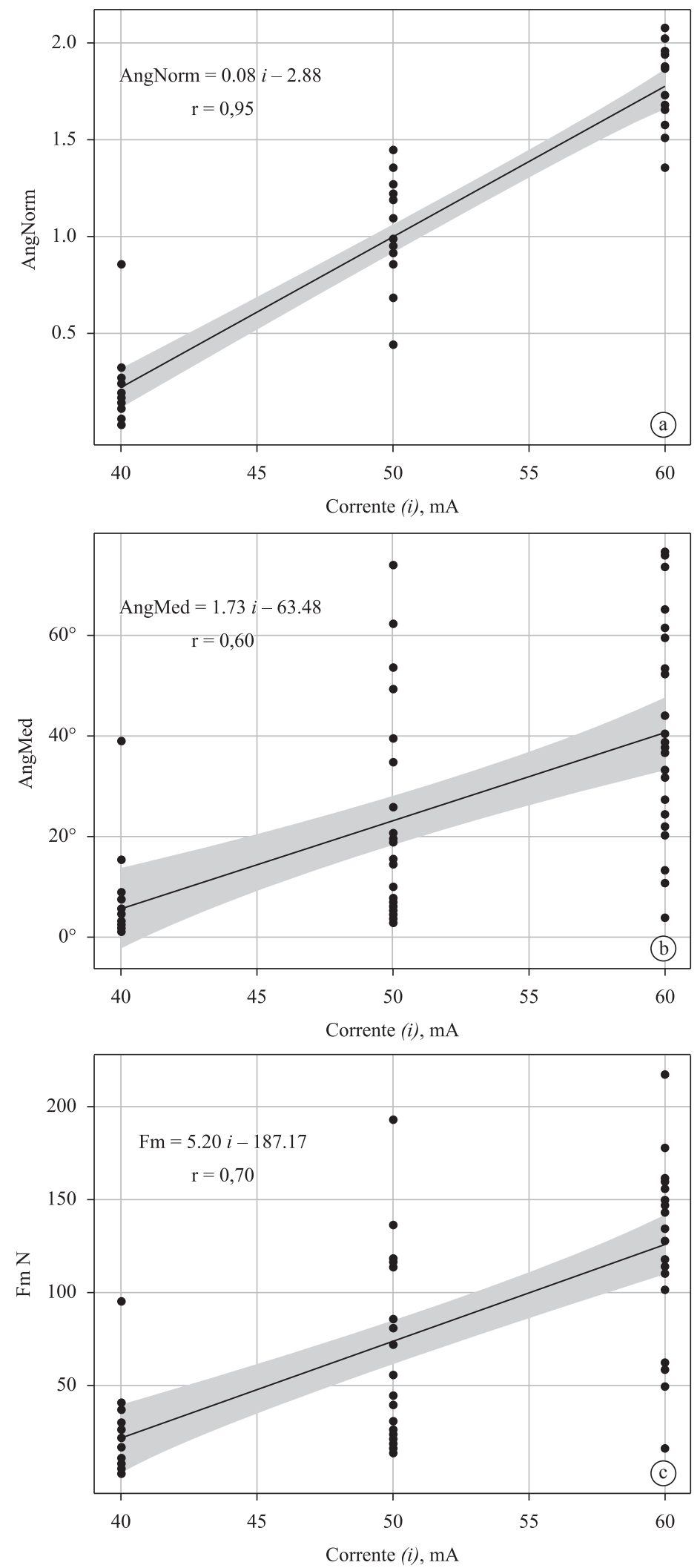

Figura 6. Retas de regressões lineares, com intervalo de confiança de $95 \%$. Frequência e duração de pulso de, respectivamente, $50 \mathrm{~Hz}$ e $300 \mu$ s. Figure 6. Linear Regression, with trust interval of $95 \%$. Frequency and pulse duration of respectively $50 \mathrm{~Hz}$ and $300 \mu \mathrm{s}$. 


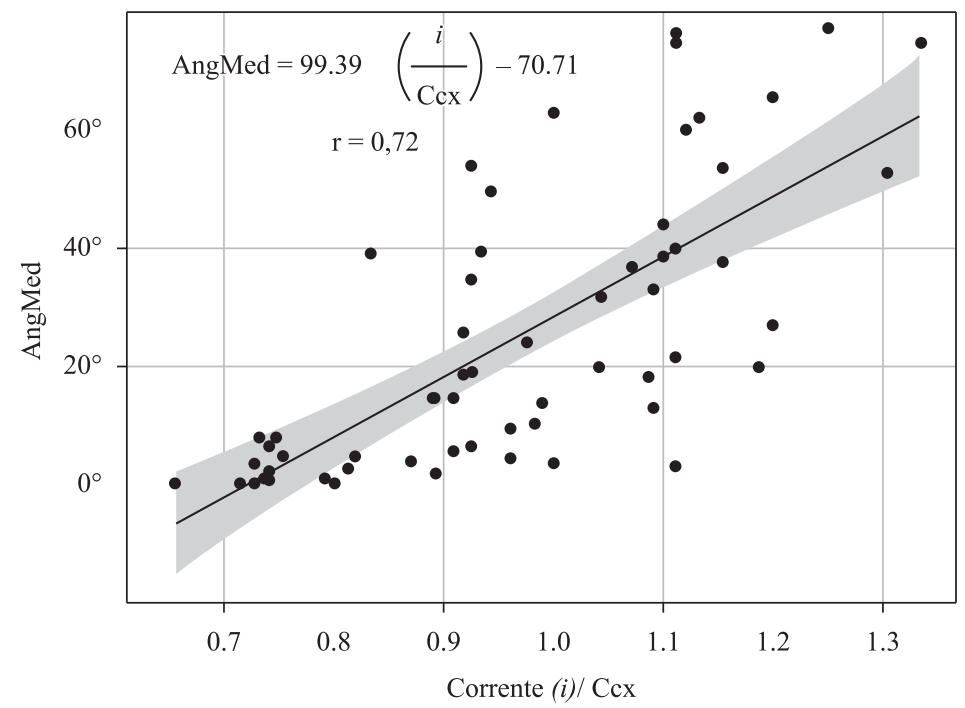

Figura 7. Reta de regressão linear do modelo da corrente normalizada pela circunferência da coxa para AngMed, ajustada com um intervalo de confiança de $95 \%$.

Figure 7: Linear Regression of the model with current normalization for the thigh length (trust interval of 95\%).

$\mathrm{Na}$ Fase II as intensidades de corrente foram preditas pelo modelo descrito pela Equação $12 \mathrm{com}$ o normalizador $C c x$. Foram preditas correntes para alcançar angulações de $15,30 \mathrm{e} 45^{\circ}$. As estimativas de corrente obtidas na Fase II foram superestimadas pela equação obtida na Fase I. Mesmo assim a modelagem realizada empregando a Equação 12, normalizador $C c x$ e dados da Fase II mostrou correlação linear de 0,798 , semelhante à encontrada na Fase I. O resultado da correlação entre as variações angulares medidas e as esperadas, na etapa de comprovação do modelo, pode ser verificado na Figura 8 .

$i=\left(\frac{\text { AngMed }+70,71}{99,39}\right) C c x$

\section{Discussão}

A aplicação clínica de tratamentos utilizando sistemas de FES requer que seja possível determinar os padrões de estimulação adequados para a realização de um movimento pré-estabelecido (Pedotti e Ferrarin, 1992; Perumal et al., 2006). O presente trabalho investigou, então, a influência de parâmetros (frequência, intensidade de corrente e duração do pulso) da FES usada no estabelecimento da posição angular e força, apresentando um modelo capaz de estimar a relação entre a corrente de estimulação e a variação angular produzida durante a extensão do joelho.

O procedimento analítico apresentou um modelo mecânico em função do ângulo e da corrente de estimulação (Equação 7), considerando o membro inferior como uma cadeia cinemática aberta composta de dois segmentos rígidos: a coxa e o complexo pernapé. Ferrarin e Pedotti (2000) também propuseram um modelo analítico para explicar características articulares em função de parâmetros da FES. No entanto estes autores utilizaram dados de difícil determinação clínica, tais como características passivas da articulação do joelho e da perna inferior, além de considerar as características gravitacionais e de inércia dos segmentos anatômicos, propriedades de amortecimento e rigidez (que consideram os componentes elástico e viscoso da articulação). Por outro lado, o modelo desenvolvido no presente trabalho buscou o uso de parâmetros facilmente mensuráveis para a estimativa da posição angular. Estes parâmetros poderiam guardar alguma relação com todos estes efeitos e outros parâmetros difíceis de serem obtidos na clínica. Alguns autores (Doheny et al., 2010; Gave et al., 2011; Maffiuletti, 2010; Maffiuletti et al., 2011; Petrofsky, 2008) já haviam apontado à necessidade de mais investigações destes parâmetros, pois há indícios de que nem toda a corrente pode ser utilizada para disparar o movimento ou que a sensibilidade individual pode afetar os resultado (Doheny et al., 2010; Maffiuletti et al., 2011; Petrofsky, 2008; Petrofsky et al., 2008). A existência de um parâmetro antropométrico capaz de melhorar a predição do movimento a partir de características individuais parece realmente existir. Em nosso trabalho foi observado uma forte correlação $(0,948)$ entre a corrente e a angulação normalizada pela média das angulações produzidas em cada voluntário (Figura 6). Se fosse possível isolar as características antropométricas que permitem esta previsão elas poderiam ser usadas na prática clínica 


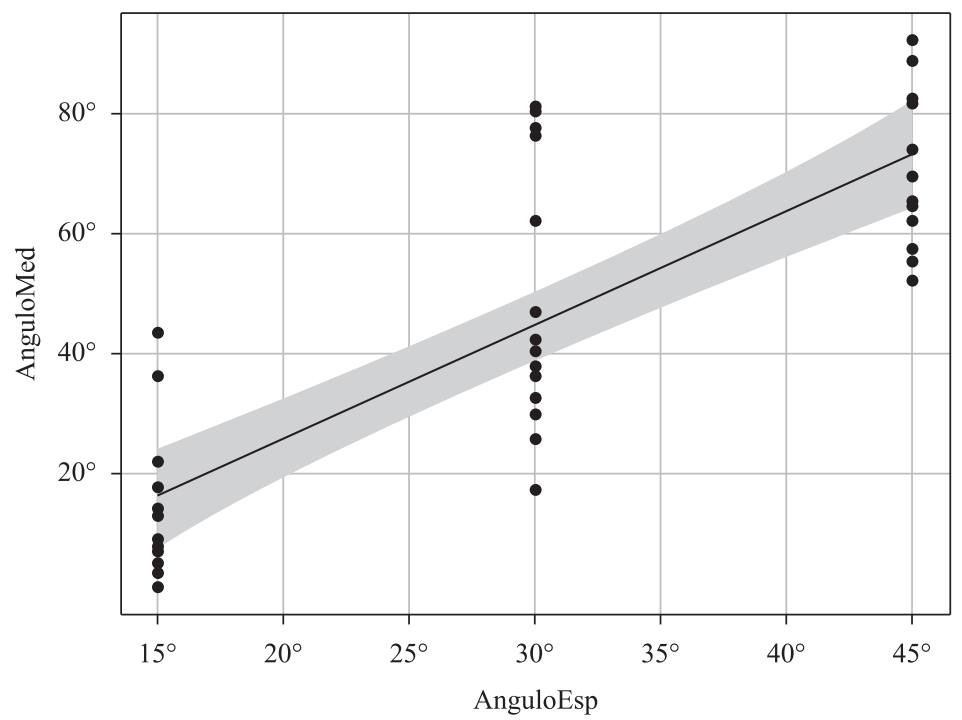

Figura 8. Gráfico da variação angular medida versus a variação angular calculada pelo modelo dado pela Equação 11. Apresenta-se nele de ajuste dos dados.

Figure 8. Angular variation measured vs Angular variation predicted (Equation 11). The grey line indicates the adjusted model.

para a determinação dos perfis de corrente usados para movimentação passiva da articulação do joelho.

Verificou-se, no presente estudo, que a frequência (Figura 5) e duração de pulso da estimulação elétrica (Tabela 2) não têm relevância estatística no comportamento da AngMed e Fm durante a extensão de joelho. Tais achados corroboram os resultados apresentados por Chou et al. (2005) que mostram forças similares produzidas para frequências de estimulação de 30,50 e $70 \mathrm{~Hz}$, mas difere dos resultados de alguns estudos apresentados na literatura (Ferrarin e Pedotti, 2000; Kramer, 1987; Perumal et al., 2006; Scheeren et al., 2010) que encontraram o torque produzido diretamente dependente da frequência da estimulação. Provavelmente os resultados apresentados nesses trabalhos diferem dos nossos devido ao fato dos autores terem usado, além de frequências mais comuns iguais ou acima de $50 \mathrm{~Hz}$, frequências de teste mais baixas $(10,20,25 \mathrm{~Hz})$ as quais não conseguiram produzir uma contração tetânica e geraram uma sensação dolorosa maior, o que provocou a diferença significativa entre a resposta para essas frequências e aquelas acima de $30 \mathrm{~Hz}$ mais próximas de, ou superiores, a frequência do tétano.

No entanto, estes últimos autores observaram um efeito de saturação para altas frequências, concordando que o aumento da frequência de estimulação além da frequência de tétano não altera a produção de forca no músculo. Fato que também foi verificado no presente trabalho (Figura 5); onde valores similares de AngMed e $\mathrm{Fm}$ foram produzidos usando-se frequências de 50 e $80 \mathrm{~Hz}$. Devido ao mencionado efeito a frequência de $50 \mathrm{~Hz}$ foi escolhida (teste de Kruskal-Wallis) dentre as três testadas $(30,50$ e $80 \mathrm{~Hz})$ para fazer parte da Fase II.

Para uma frequência e duração de pulso fixas em $50 \mathrm{~Hz}$ e $300 \mu \mathrm{s}$, respectivamente, observou-se uma relação direta do aumento da amplitude de corrente com o incremento de AngMed ( $r=0,706)$ e Fm $(r=0,701)$. Lynch e Popovic (2011) também observaram diferenças estatisticamente significativas das variações angulares da articulação do joelho com diferentes amplitudes da intensidade de corrente estimulando o músculo vasto lateral, o mesmo músculo escolhido para nosso protocolo por prover um movimento de joelho anatomicamente correto, sem adução.

O modelo apresentado por El Makssoud et al. (2003) mostra a existência de relação entre variáveis antropométricas e a força muscular produzida por eletroestimulação. Gave et al. (2011) relatam que o uso dessas variáveis pode ajudar na estimativa da intensidade da corrente de estimulação para produzir uma variação angular pré-estabelecida. No estudo da influência de algumas variáveis antropométricas, o presente trabalho observou que a melhor correlação entre a corrente de estimulação e o ângulo articular foi obtida normalizando-se a intensidade pela circunferência da coxa $(r=0,723)$. Do mesmo modo, para a correlação entre a corrente de estimulação e a força muscular estimada, obteve-se o melhor resultado com a normalização da intensidade pela circunferência da coxa $(r=0,685)$, apesar do uso deste 
normalizador piorar a qualidade da estimativa. O estudo de Gave et al. (2011) corrobora os resultados obtidos e o uso da circunferência do segmento estimulado como um possível normalizador da corrente.

No entanto, alguns estudos afirmam que o tecido gorduroso interfere na propagação da corrente para o músculo (Doheny et al., 2010; Maffiuletti et al., 2011; Petrofsky, 2008; Petrofsky et al., 2008), sugerindo a dobra cutânea, a área de gordura ou, inclusive, o índice de massa corporal como melhores normalizadores para modelos de predição da resposta individual sensorial e motora. Fato este que diverge dos nossos resultados, mas que pode explicar a forte correlação encontrada entre a corrente normalizada pela área transversal da coxa (dado calculado levando em conta o valor da área de gordura do segmento) e a variação angular produzida durante a extensão de joelho. A divergência de resultados pode ser explicada pelas diferenças nos protocolos experimentais usados por Doheny et al. (2010), Maffiuletti et al. (2011) e Petrofsky (2008) que, por exemplo, se limitam a uma análise atingindo o limiar motor ou com uma mínima movimentação do membro. Merletti et al. (2010) usou eletrodos com áreas diferentes e de agulha, para minimizar a perda de efetividade da eletroestimulação devida à mudança considerável na zona de inervação muscular que acontece por pequenas variações articulares.

Contudo, na literatura é sugerido que melhores modelos de predição podem ser alcançados estudandose outras características do indivíduo, além das antropométricas, Exemplos comuns são: o limiar de disparo motor, o nível de fadiga, o fluxo sanguíneo cutâneo na área estimulada ou determinando a influência do sexo nos efeitos da FES (Kramer, 1987; Lynch e Popovic, 2011; Maffiuletti et al., 2008, 2011; Petrofsky et al., 2008). Acredita-se que estas variáveis, se inseridas na modelagem proposta, poderiam aproximar mais os valores experimentais da variação do ângulo da articulação do joelho daqueles pré-determinados ou desejados.

\section{Agradecimentos}

Os autores agradecem à CAPES, ao CNPQ e o convênio PEC-PG pelo apoio financeiro.

\section{Referências}

Binder-Macleod SA, Lee SCK. Assessment of the efficacy of functional electrical stimulation in patients with hemiplegia. Topics in Stroke Rehabilitation. 1997; 3:88-98.

Braz GP, Russold M, Davis GM. Functional electrical stimulation control of standing and stepping after spinal cord injury: A review of technical characteristics. Neuromodulation: Technology at the Neural Interface. 2009; 12(3):180-90.
PMid:22151359. http://dx.doi.org/10.1111/j.15251403.2009.00213.x

Chou L, Ding J, Wexler AS, Binder-Macleod SA. Predicting optimal electrical stimulation for repetitive human muscle activation. Journal of Electromyography and Kinesiology. 2005; 15(3):300-9. PMid:15763677. http:// dx.doi.org/10.1016/j.jelekin.2004.10.002

Doheny EP, Caulfield BM, Minogue CM, Lowery MM. Effect of subcutaneous fat thickness and surface electrode configuration during neuromuscular electrical stimulation. Medical Engineering \& Physics. 2010; 32(5):468-74. PMid:20417145. http://dx.doi.org/10.1016/j. medengphy.2010.03.004

El Makssoud H, Poignet P, Guiraud D. Modelling of the skeletal muscle under functional electrical stimulation. In: IFESS'03: Proceedings of the Annual Conference of theInternational Functional Electrical Stimulation Society; 2003; Sunshine Coast. Sunshine Coast; 2003.

Enoka RM. Neuromechanical basis of kinesiology. 2nd ed. Human Kinetics Publishers; 1994.

Ferrarin M, Pedotti A. The relationship between electrical stimulus and joint torque: a dynamic model. IEEE Transactions on Rehabilitation Engineering. 2000; 8(3):342-52. http:// dx.doi.org/10.1109/86.867876

Franken HM, Veltink PH, Tijsmans R, Nijmeijer H, Boom HBK. Identification of passive knee joint and shank dynamics in paraplegics using quadriceps stimulation. IEEE Transactions on Rehabilitation Engineering. 1993; 1(3):154-64. http://dx.doi.org/10.1109/86.279264

Gave NS, Souza MN, Pino AV. Estudo da influência de variáveis antropométricas nos efeitos da estimulação elétrica funcional com diferentes intensidades de estímulo [dissertação]. Rio de Janeiro: Universidade Federal do Rio de Janeiro; 2011.

Guedes DP. Manual prático para avaliação em educação física. Editora Manole Ltda; 2006.

Ihaka R, Gentleman R. R: a language for data analysis and graphics. Journal of Computational and Graphical Statistics. 1996; 5(3):299-314.

Kitchen S. Eletroterapia: prática baseada em evidências. 11. ed. Manole; 2003.

Kramer JF. Effect of Electrical Stimulation Current Frequencies on Isometric Knee Extension Torque. Physical Therapy. 1987; 67(1):31-8. PMid:3797474.

Lynch CL, Graham GM, Popovic MR. A generic model of real-world non-ideal behaviour of FES-induced muscle contractions: simulation tool. Journal of Neural Engineering. 2011; 8(4):046034. PMid:21757801. http:// dx.doi.org/10.1088/1741-2560/8/4/046034

Lynch CL, Popovic MR. A stochastic model of knee angle in response to electrical stimulation of the quadriceps and hamstrings muscles. Artificial Organs. 2011; 35(12):1169-74. PMid:21810111. http://dx.doi.org/10.1111/j.15251594.2011.01228.x

Maffiuletti NA. Physiological and methodological considerations for the use of neuromuscular 
electrical stimulation. European Journal of Applied Physiology. 2010; 110(2):223-34. PMid:20473619. http:// dx.doi.org/10.1007/s00421-010-1502-y

Maffiuletti NA, Herrero AJ, Jubeau M, Impellizzeri FM, Bizzini M. Differences in electrical stimulation thresholds between men and women. Annals of Neurology. 2008; 63(4):507-12. PMid:18300313. http:// dx.doi.org/10.1002/ana.21346

Maffiuletti NA, Morelli A, Martin A. Effect of gender and obesity on electrical current thresholds. Muscle \& Nerve. 2011; 44(2):202-7. PMid:21698650. http://dx.doi. org/10.1002/mus.22050

Merletti R, Aventaggiato M, Botter A, Holobar A, Marateb $\mathrm{H}$, Vieira T. Advances in surface EMG: recent progress in detection and processing techniques. Critical Reviews in Biomedical Engineering. 2010; 38(4): 305-45. http://dx.doi. org/10.1615/CritRevBiomedEng.v38.i4.10

Mohammed S, Poignet P, Fraisse P, Guiraud D. Optimal stimulation patterns for knee joint movement restoration during co-contraction of antagonist muscles. In: Biomedical Robotics and Biomechatronics (BioRob): Proceedins of the 3rd IEEE RAS and EMBS International Conference on Biomedical Robotics and Biomechatronics (BioRob); 2010; Tokyo. IEEE; 2010. p. 687-92. http://dx.doi.org/10.1109/ BIOROB.2010.5626111

Peckham PH, Knutson JS. Functional electrical stimulation for neuromuscular applications. Annual Review of Biomedical Engineering. 2005; 7(1):327-60. PMid:16004574. http:// dx.doi.org/10.1146/annurev.bioeng.6.040803.140103

Pedotti A, Ferrarin M. Restoration of walking for paraplegics: recent advances and trends. IOS Press; 1992.

Perumal R, Wexler AS, Binder-Macleod SA. Mathematical model that predicts lower leg motion in response to electrical stimulation. Journal of Biomechanics. 2006; 39(15):2826-36. PMid:16307749. http://dx.doi.org/10.1016/j. jbiomech.2005.09.021

Petrofsky J. The effect of the subcutaneous fat on the transfer of current through skin and into muscle. Medical Engineering
\& Physics. 2008; 30(9):1168-76. PMid:18400550. http:// dx.doi.org/10.1016/j.medengphy.2008.02.009

Petrofsky JS, Suh HJ, Gunda S, Prowse M, Batt J. Interrelationships between body fat and skin blood flow and the current required for electrical stimulation of human muscle. Medical Engineering \& Physics. 2008; 30(7):931-6. PMid:18243763. http://dx.doi.org/10.1016/j. medengphy.2007.12.007

Pino AV, Costa JCGD, Jandre FC, Giannella A. Mecânica - um programa para análise do sistema respiratório. In: Congresso Brasileiro de Engenharia Biomédica: Anais do XVIII Congresso Brasileiro de Engenharia Biomédica; 2002; São José dos Campos. São José dos Campos; 2002.

Pino AV, Kagami LT, Jandre FC, Giannella A. DAS - Um programa de aquisição e processamento de sinais para engenharia pulmonar. In: III CLAEB: Anais do $3^{\circ}$ Congresso Latino Americano de Engenharia Biomédica; 2004; João Pessoa. João Pessoa; 2004. p.765-8.

Scheeren ME, Nogueira-Neto NG, Krueger-Beck E, Button VLSN, Nohama P. Investigation of muscle behavior during different functional electrical stimulation profiles using mechanomyography. Conference proceedings Annual International Conference of the IEEE Engineering in Medicine and Biology Society. 2010; 2010:3970-3. http:// dx.doi.org/10.1109/IEMBS.2010.5627986

Singer B. Functional electrical stimulation of the extremities in the neurological patient: a review. The Australian Journal of Physiotherapy. 1987; 33(1):33.

Sinkjaer T, Popovic DB. Neurorehabilitation technologies Present and future possibilities. NeuroRehabilitation. 2009; 25(1):1-3.

Tozeren A. Human body dynamics - classical mechanics and human movement. Springer; 2000.

Velloso JB. Sistema para estimulação elétrica funcional (FES-PEB) [dissertação]. Rio de Janeiro: Universidade Federal do Rio de Janeiro; 2007.

\footnotetext{
Autores

Ingrid Johanna Rodríguez Bohórquez*

Universidade Federal do Rio de Janeiro - UFRJ, Rio de Janeiro, RJ, Brasil. 